\title{
A Risk Assessment Model for Dam Combining the Probabilistic and the Nonprobabilistic Methods
}

\author{
Yantao Zhu $\mathbb{D}^{1,2}$ Xinqiang Niu, ${ }^{3,4}$ Jimin Wang, ${ }^{5}$ Chongshi Gu $\mathbb{D}^{1,2}$ Qiang Sun, ${ }^{6}$ Bo Li, ${ }^{1,2}$ \\ and Lixian Huang ${ }^{7}$ \\ ${ }^{1}$ State Key Laboratory of Hydrology-Water Resources and Hydraulic Engineering, Hohai University, Nanjing, China \\ ${ }^{2}$ College of Water Conservancy and Hydropower Engineering, Hohai University, Nanjing, China \\ ${ }^{3}$ Changjiang Institute of Survey, Planning, Design and Research, Wuhan, China \\ ${ }^{4}$ National Dam Safety Research Center, Wuhan, China \\ ${ }^{5}$ Yalong River Hydropower Development Company, Ltd., Chengdu 610051, China \\ ${ }^{6}$ CCCC Third Harbor Engineering Co.Ltd., Shanghai, China \\ ${ }^{7}$ Materials Science and Engineering, University of California-Los Angeles, Los Angeles, CA 90095, USA
}

Correspondence should be addressed to Yantao Zhu; zyt50@163.com and Chongshi Gu; csgu@hhu.edu.cn

Received 27 January 2020; Revised 12 March 2020; Accepted 18 March 2020; Published 27 April 2020

Academic Editor: Ioannis Kostavelis

Copyright (c) 2020 Yantao Zhu et al. This is an open access article distributed under the Creative Commons Attribution License, which permits unrestricted use, distribution, and reproduction in any medium, provided the original work is properly cited.

The dam reliability study is essential for dam operation safety, regarding the complexity in dam failure causes. The assessment of the dam reliability is now mainly probabilistic or nonprobabilistic. The probabilistic method is usually applied to the cases with sufficient knowledge on dam parameters, while the nonprobabilistic method is suitable for the cases with insufficient knowledge on dam parameters. Since a dam can contain multiple parameters, information abundancy can vary among those parameters, and neither the probabilistic method nor the nonprobabilistic method alone is enough for dam reliability assessment. In this paper, the probabilistic method and nonprobabilistic method are modified based on the adjusted first-order second-moment method and the interval analysis method to suit the dam reliability assessment. Based on characterization on these two methods and the research of the fusion method, the secondary performance function of the dam is constructed, and the construction method of the risk assessment model for dam is proposed. Combined with a case study, this paper contributes to the safe operation of the dam.

\section{Introduction}

The risk probability of a dam, which is the calculation of the possibility of the risk path failure, is an important part of dam risk management. Internationally, scholars have studied the evaluation method of the risk probability from probabilistic and nonprobabilistic aspects.

1.1. Probabilistic Risk Assessment Methods. Malkawi et al. [1] established performance functions based on the Swedish circle method, simplified Bishop's method, simplified Janbu's method, and Spencer's method, respectively. The firstorder second-moment method and the Monte Carlo simulation method were used to solve the reliability index, and the instability reliability model was established based on the reliability index. Kruger et al. [2] calculated the risk probability of the RCC gravity dam by using the first-order second-moment method, improved first-order secondmoment method, and Monte Carlo simulation method and provided suggestions for maintenance and reinforcement of the dam based on the calculation results. Leszek [3] believed that there were various loads in the dam structure that maintained or caused the crash, and the uncertainty of the load ratio could be estimated. Based on this load ratio, the probability of dam instability was calculated. Leszek [4] assumed that the antisliding and sliding forces were controlled within the accuracy range of $15 \%$. Moreover, the 
probability of hydraulic structures instability risk was calculated by using the additional error principle and Gaussian distribution. During seismic events of the Kajiwa concreteface rock-fill dam, Liang et al. [5] directly established the iterative formula of checking points in generalized stochastic space, so as to solve the problem of calculating the structural risk probability with related random variables conveniently. $\mathrm{Ma}$ [6] calculated the reliability of the horizontal displacement of the dam crest with the checking point method and took the elastic modulus of concrete as the random variable in three different sections of the gravity dam. Zheng et al. [7] believed that the correlation of random variables conformed to the Nataf distribution, so they applied the Monte Carlo simulation method of Cholesky decomposition to calculate the probability of collapse of tailings dam slope. Liu et al. [8] applied the finite element method (FEM), support vector machine (SVM), and Monte Carlo simulation method to calculate the accident risk probability, which greatly reduced the calculation time and workload with good accuracy. Based on the rigid limit equilibrium safety factor method, Wang et al. [9] proposed the particle swarm optimization algorithm to analyze the stability reliability of multislip surface of gravity dam foundation and verified that the method is highly practical. Wang et al. [10] proposed a method to calculate the antislip stability reliability of gravity dam foundation based on genetic algorithm, which overcame the limitation that the function needs to be expressed as an explicit function and enriched the applicability of the first-order second-moment method in the current design standards and specifications. Their method can be used for the research of antislip stability reliability of gravity dam foundation under the condition of multiple sliding surfaces. Lai et al. [11] proposed the partial coefficient method of bounded strength drop, analyzed the stability of deep antislip, and verified that their method was reasonable and feasible. Hariri-Ardebil [12] proposed a method to calculate the probability of concrete dam reliability based on nonlinear finite element analysis using the mixed parameter probabilistic statistics method.

1.2. Nonprobabilistic Risk Assessment Methods. In the case of insufficient engineering data, Elishakoff [13] used the convex model for uncertainty analysis and verified the effectiveness of the method. Ben-Haim [14] considered the uncertainty of the variables and verified that the variation of the variables within a certain range had no great influences on the structure reliability. Elishakoff [15] raised the idea that the nonprobabilistic reliability index should be a specific interval rather than a value. Based on the properties of the convex set operation and considering the maximum allowable uncertainty of the system, Ben-Haim [16] proposed a theory of the nonprobabilistic reliability. Pantelides and Ganzali $[17,18]$ established the ellipsoidal convex model and the uniformly bounded convex model and applied these two models to the engineering design optimization. Then, the validity of the two models is verified in comparison with the worst-case design. Yuan et al. [19] put forward the nonprobabilistic reliability method to establish the reliability model of gravity dam and bedrock index and discussed the probabilistic reliability index of the gravity dam stability against sliding failure mode. Zhang [20] proposed the multidimensional parallelepiped nonprobabilistic model which can effectively deal with complex uncertainty problems of the coexisting related variables and independent variables. Liu [21] considered the uncertainty caused by the interval of the nonlinear system in the nonprobabilistic reliability, calculated the reliability with the grey number, and successfully overcame the influence of the interval operation uncertainty. $\mathrm{Su}$ and Hao [22] proposed the dynamic response surface method of Gaussian process for particle swarm optimization to calculate the nonprobabilistic reliability analysis of complex engineering structures. Meanwhile, they analyzed and proved that the Gaussian process dynamic response surface method of particle swarm optimization is more accurate. Aiming to solve the problem of insufficient uncertain parameters, Liang [23] proposed an interval analysis method to solve the problem of parameter uncertainty in the structure and established the index of nonprobabilistic reliability analysis. To overcome the poor engineering applicability of the traditional reliability analysis method based on the convex model, Bi [24] developed a new simulation algorithm to match the reliability analysis model and established two first-order approximate reliability analysis methods. Yun et al. [25] combined the improved Kriging model with the finite element method to establish a nonprobabilistic reliability model of the beam structure resonance with a superellipsoid convex set. Zheng et al. [26] used the SVM method to reselect and construct the response surface function and used random variables with statistical parameters to obtain the structure reliability. Sun et al. [27] constructed an explicit quadratic response surface function and combined it with the structural nonprobabilistic model to analyze the stability of an engineering structure. Li [28] proposed a time-varying reliability index model from the interval nonprobabilistic model, considering the influence of structural resistance with time.

1.3. Motivation. In the probabilistic risk assessment method, the nonlinearity of the limit state function of the dam is generally high, so the accuracy of analysis results are frequently affected by random factors. In this case, the probability distribution and the digital characteristics of the random factors can severely influence the assessment accuracy. As a result, the traditional random impact factor distribution type of the method has certain subjectivity that decreases the assessment precision.

Meanwhile, the nonprobabilistic risk assessment method is less applicable to the situation where the statistical data of the impact factors are deficient and the fluctuation interval is the only known factor. Unfortunately, few application cases of the nonprobabilistic risk assessment method in the safety assessment of dam exist, and usually the correlation between factors is not considered.

A dam contains various parameters, so information abundancy can be unbalanced. Neither a single probabilistic model nor a single nonprobabilistic model is suitable for the 
dam failure risk assessment. As a result, a combination of probabilistic model and nonprobabilistic model should be studied to solve the risk analysis of dam failure under different operation conditions.

\section{A Probabilistic Risk Assessment Model for Dams}

At present, the calculation methods for dam reliability evaluation [29-32] are as follows: average first-order secondmoment method, second-order second-moment method, response surface method, direct integration method, Monte Carlo simulation method, and Latin hypercube sample method. The next part focuses on the adjusted first-order second-moment method. The corresponding probability assessment model for dam reliability evaluation is established.

2.1. A Characterization Method of the Probabilistic Risk Assessment Model for Dams. The limit state of the dam can be described by the performance function, which can be expressed as

$$
g(X)=g\left(x_{1}, x_{2}, \ldots, x_{n}\right),
$$

where $X=\left(x_{1}, x_{2}, \ldots, x_{n}\right)$ is a set of basic variables and $x_{i}\left(i=1,2, \ldots, x_{n}\right)$ is the dam structure factor variables.

The function can be summarized in the following three forms: (1) when $g(X)=0$, it means that the dam structure reaches a limit state which is also easily changed; (2) when $g(X)<0$, the dam structure is in a state of failure, and the structure cannot function correspondingly. The failure state is only theoretical and not realistic because once $g(X)<0$, the structure of the dam shifts until $g(X) \geq 0$; (3) when $g(X)>0$, it indicates that the structure is in a safe state, and the structure can function correspondingly. Generally, the dam structure is in the safe state.

Suppose the dam structure has potential risks, which means the dam may not complete its function correspondingly under the structure safety. Consequently, the multidimensional integral expression of the corresponding risk probability is

$$
P_{f}=\int_{\Omega} f\left(x_{1}, x_{2}, \ldots, x_{n}\right) \mathrm{d} x_{1} \mathrm{~d} x_{2} \cdots \mathrm{d} x_{n},
$$

where $P_{\mathrm{f}}$ is the risk probability; $\Omega$ is the failover domain; and $f\left(x_{1}, x_{2}, \ldots, x_{n}\right)$ is the joint probability density function of the vector $X$.

For the dam engineering, it is difficult to calculate the risk probability index by equation (2) in a probabilistic method. Usually, the dam reliability is calculated first, and the probabilistic reliability index is converted into the risk probability. Finally, the limit state function of the dam is expressed as the probabilistic reliability index:

$$
\beta=\frac{\mu_{g}}{\sigma_{g}}
$$

where $\mu_{g}$ is the mean of the function $g(X)$ and $\sigma_{g}$ is the standard deviation of the function $g(X)$.
2.2. An Adjusted First-Order Second-Moment Method. In the dam reliability analysis, the first-order second-moment center point method needs the mean and the standard deviation values of the mathematical model to calculate the reliability of dam structure. The limit state performance function $Z=g\left(x_{1}, x_{2}, \ldots, x_{n}\right)$ of the dam is expanded by Taylor series at the point of mean value and linearized the expanded function to solve the dam reliability.

When calculating the probabilistic reliability index using the basic first-order second-moment method, two kinds of problem will exist: (1) when the nonlinear function is expanded by Taylor series and the corresponding higher order terms are omitted, the error increases along with the distance between some points and the limit state surface. This phenomenon results from the average point specific location, which is generally in the reliable region, not on the limit surface. (2) When selecting different performance functions, the values vary with meanings, and the huge error values provide different reliable indexes. Therefore, the basic firstorder second-moment method is difficult to meet the requirements of engineering application.

In view of the deficiency in the basic first-order secondmoment method, the following is an improvement of the basic first-order second-moment method. The basic idea is as follows: the probability function is expanded by Taylor series, and the high-order nonlinear parts are replaced by the linear expressions, while the lower order terms stay the same. The mean and variance values of the parameter variables can plug in the linear expression. This method can help to solve the nonlinear problem of the performance equation effectively and analyze the influence of the mean and variance on the dam reliability.

Suppose the limit state equation of the dam is

$$
Z=g(X)=0 \text {. }
$$

Assume $x^{*}=\left(x_{1}^{*}, x_{2}^{*}, \ldots, x_{n}^{*}\right)^{T}$ is a point on the limit state surface of the dam structure, namely,

$$
g\left(x^{*}\right)=0 .
$$

The limit state equation of the dam is expanded in Taylor series at point $x^{*}$, and the first-order part is taken:

$$
Z_{L}=g\left(x^{*}\right)+\sum_{i=1}^{n} \frac{\partial g\left(x^{*}\right)}{\partial X_{i}}\left(X_{i}-x_{i}^{*}\right) .
$$

In the variable factor $X$ space, $Z_{L}=0$ is the tangent plane of the limit state plane passing $x^{*}$, and the mean and the standard deviation values of $Z_{L}$ are as follows:

$$
\begin{aligned}
\mu_{Z_{L}} & =g\left(x^{*}\right)+\sum_{i=1}^{n} \frac{\partial g\left(x^{*}\right)}{\partial X_{i}}\left(\mu_{X_{i}}-x_{i}^{*}\right), \\
\sigma_{Z_{L}} & =\sqrt{\sum_{i=1}^{n}\left[\frac{\partial g\left(x^{*}\right)}{\partial X_{i}}\right]^{2} \sigma_{X_{i}}^{2} .}
\end{aligned}
$$

Substituting equations (7) and (8) into equation (3), the probabilistic reliability index of the dam can be calculated as 


$$
\beta=\frac{\mu_{Z_{L}}}{\sigma_{Z_{L}}}=\frac{g\left(x^{*}\right)+\sum_{i=1}^{n}\left(\partial g\left(x^{*}\right) / \partial X_{i}\right)\left(\mu_{X_{i}}-x_{i}^{*}\right)}{\sqrt{\sum_{i=1}^{n}\left[\partial g\left(x^{*}\right) / \partial X_{i}\right]^{2} \sigma_{X_{i}}^{2}}} .
$$

For the convenience of calculation, $X_{i}$ is transformed into the standard normal variable factor $Y$ space, that is, $Y_{i}=\left(X_{i}-\mu_{X_{i}}\right) / \sigma_{X_{i}}$. Therefore, the probabilistic reliability index is expressed as

$$
\beta=\frac{\mu_{Z_{L}}}{\sigma_{Z_{L}}}=-\frac{\sum_{i=1}^{n}\left(\partial g\left(x^{*}\right) / \partial X_{i}\right) \sigma_{X_{i}}}{\sqrt{\sum_{i=1}^{n}\left[\partial g\left(x^{*}\right) / \partial X_{i}\right]^{2} \sigma_{X_{i}}^{2}}} Y_{i} .
$$

According to equation (10), the sensitivity coefficients of $X_{i}$ and $Y_{i}$ can be obtained as

$$
\alpha_{X_{i}}=\cos \theta_{X_{i}}=\cos \theta_{Y_{i}}=-\frac{\sum_{i=1}^{n}\left(\partial g\left(x^{*}\right) / \partial X_{i}\right) \sigma_{X_{i}}}{\sqrt{\sum_{i=1}^{n}\left[\partial g\left(x^{*}\right) / \partial X_{i}\right]^{2} \sigma_{X_{i}}^{2}}} .
$$

Through equation (11), the expression of the transformation of the design check point to the standard normal $Y$ space is

$$
y_{i}^{*}=\beta \cos \theta_{Y_{i}}, \quad i=1,2, \ldots, n,
$$

Substitute the results back to $X$ space. Then,

$$
x_{i}^{*}=\mu_{x_{i}}+\beta \sigma_{X_{i}} \cos \theta_{X_{i}}, \quad i=1,2, \ldots, n .
$$

From the theoretical calculation methods mentioned above, combining equations (6), (10), (11), and (13) [33, 34], we can get the probabilistic reliability indexes $\beta$ and $x^{*}$ based on HL-RF iterative solution whose formula is shown in the following equation:

$$
X_{K+1}=\frac{1}{\left|\nabla g\left(X_{k}\right)\right|^{2}}\left[\nabla g\left(X_{k}\right) X_{k}-g\left(X_{k}\right)\right] \nabla g\left(X_{k}\right)^{T} .
$$

2.3. A Calculation Model for Dam Reliability. The probabilistic reliability index is solved by an adjusted first-order second-moment method, and the risk probability is obtained. Therefore, based on the probabilistic reliability index model, the relationship between the reliability and the risk probability is established to construct the calculation model for the dam reliability.

The probabilistic reliability index expression of equation (10) shows that the higher the probabilistic reliability index $\beta$, the lower the risk probability of dam failure and the other way around. Therefore, when the variable factors in the performance function $g(X)=g\left(x_{1}, x_{2}, \ldots, x_{n}\right)$ conform to a normal distribution, or when the nonnormal variable factors have been normalized and the variables are independent of each other, the performance function $g(X)$ also conforms to a normal distribution. In this case, the relationship between the probabilistic reliability index and the risk probability of the dam can be deduced as follows:

$$
p_{f}=\Phi(-\beta) \text {. }
$$

2.4. Effects of the Mean and Variance of Dam Parameters on the Values of the Risk Probability. From equation (9), the probabilistic reliability index can be derived from the mean and variance of parameter variables. Since the environment of a dam system is complex and changeable, and the operation life length of a dam is long, the mean and variance will inevitably fluctuate in some extent, which will affect the risk probability value of dam accidents. Therefore, it is necessary to study the influence of the mean and variance of dam parameters on the values of risk probability.

In view of the problems mentioned above, since the risk probability is calculated by the probabilistic reliability index in the probabilistic assessment model for dam reliability evaluation, the influence of the volatility of the mean and variance of the dam parameters on the probabilistic reliability index should be analyzed first.

Based on equation (9), the partial derivatives of the reliability index with respect to $\mu_{X_{i}}$ and $\sigma_{X_{i}}$, respectively, can be obtained by

$$
\begin{aligned}
& a=\frac{\partial \beta}{\partial \mu_{X_{i}}}=\frac{\left(\partial g\left(x^{*}\right) / \partial X_{i}\right) \mu_{X_{i}}}{\sqrt{\sum_{i=1}^{n}\left[\partial g\left(x^{*}\right) / \partial X_{i}\right]^{2} \sigma_{X_{i}}^{2}}} \\
& b=\frac{\partial \beta}{\partial \sigma_{X_{i}}}=-\frac{\left[g\left(x^{*}\right)+\sum_{i=1}^{n}\left(\partial g\left(x^{*}\right) / \partial X_{i}\right)\left(\mu_{X_{i}}-x_{i}^{*}\right)\right] \sigma_{X_{i}}}{\left(\sum_{i=1}^{n}\left[\partial g\left(x^{*}\right) / \partial X_{i}\right]^{2} \sigma_{X_{i}}^{2}\right)^{3 / 2}}
\end{aligned}
$$

From equations (3) and (4), the fluctuation in the mean and variance values has a complex influence on the value of probabilistic reliability index. The results of $a$ and $b$ should be calculated from the parameter intervals of the dam variables, so the cases vary with the actual project details.

2.5. Calculation Steps of Probabilistic Risk Assessment Model for Dams. Based on the theoretical elaboration mentioned above, the following steps are summarized to compute the probabilistic risk assessment model for dams:

Step 1. To determine the limit state equation of the dam and assume that $x^{*}=\left(x_{1}^{*}, x_{2}^{*}, \ldots, x_{n}^{*}\right)^{T}$ is a point on the limit state surface

Step 2. Obtain the sensitivity coefficient of reliability function expression

Step 3. Expand the limit state equation by Taylor series at the point $x^{*}=\left(x_{1}^{*}, x_{2}^{*}, \ldots, x_{n}^{*}\right)^{T}$ and calculate its mean value and standard deviation to construct the probabilistic reliable index expression

Step 4. Calculate the probabilistic reliability index $\beta$

Step 5. If the design precision is not satisfied, the obtained $x^{*}$ value is substituted into the limit state equation and a new $x^{*}$ value should be recalculated

Step 6. Repeat Step 2 to Step 5 until the difference between the new and the previous $x^{*}$ values is less than the allowable error $\varepsilon$

Step 7. Calculate the dam reliability from the calculated value $\beta$ 
Figure 1 illustrates the calculation flow of the probabilistic assessment model for dam reliability evaluation.

\section{A Nonprobabilistic Risk Assessment Model for Dams}

When the information of dam impact factors is insufficient, it is difficult to use probabilistic risk assessment model to evaluate the dam reliability. Therefore, it is necessary to build the nonprobabilistic risk assessment model for dam. In this section, the interval analysis method is used to analyze the relationship between the nonprobabilistic reliable index and the risk probability of dam failure, so as to establish a nonprobabilistic risk assessment model for dam.

\subsection{Model Characterization of a Nonprobabilistic Risk As-} sessment Model for Dams. Ben-Haim and Elishakoff [35] proposed the concept of nonprobabilistic reliability based on the word "reliability," and believed that the smaller the range of system performance fluctuation, the more reliable the system will be. However, they did not provide a unified form of nonprobabilistic reliability index. Reference [36] established the nonprobabilistic model and gave the measurement form of the unified nonprobabilistic reliable index. Based on the abovementioned literature, this section presents a nonprobabilistic risk assessment model for the dam reliability evaluation. Suppose the dam structure performance function is

$$
Z=g\left(X_{1}, X_{2}, \ldots, X_{n}\right)=g(R, S)=R-S,
$$

where $Z$ is the structural performance function; $R$ is the uncertainty factor of resistance; and $S$ is the load uncertainty factor.

Assume the nonprobabilistic measurement of the degree of stability and reliability of dam structure is

$$
\eta=\min \left\{\|\delta\|_{\infty}\right\} \text {, }
$$

where $\eta$ is the nonprobabilistic reliability index of dam structure; $\delta$ is the normalized vector corresponding to the nonprobabilistic factor $X$; and $\|\delta\|_{\infty}$ is the infinite norm of $\delta$.

If $Z$ is the interval variable, equation (18) can be rewritten as

$$
\eta=\frac{Z^{c}}{Z^{r}}
$$

where $Z^{c}$ and $Z^{r}$ are the mean and deviation of the performance function, respectively, and can be calculated by the following formula:

$$
\begin{aligned}
& Z^{c}=\frac{Z^{l}+Z^{u}}{2}, \\
& Z^{r}=\frac{Z^{u}-Z^{l}}{2},
\end{aligned}
$$

where $Z^{u}$ and $Z^{l}$ are the maximum and minimum of the performance function $Z$, respectively.

Substitute equations (20) and (21) into equation (19) to obtain

$$
\eta=\frac{Z^{l}+Z^{u}}{Z^{u}-Z^{l}}
$$

The nonprobabilistic index $\eta$ of risk probability represents the expansion space of the interval variable that is the shortest distance from the origin of coordinates to the failure curve or surface. The higher value of $\eta$ means the stronger the safety performance of the dam structure and the lower the risk probability. Even though $Z$ value can be negative in theory, practically the state of the dam and the performance function will change when $Z<0$, which leads to the reevaluation of the $Z$ value. Therefore, as can be seen from equation (22), when $\eta>1$, both $Z^{l}$ and $Z^{u}$ are greater than 0 and $g(X)>0$, so the dam is in a safe state; when $\eta<1$, $g(X)<0$ and the dam is in a state of failure.

3.2. An Interval Analysis Method. The interval analysis method [37] is usually used to deal with the problems where the original data are not accurate, but the values of the data fall into a certain range, so the problems can have exact solutions or interval solutions.

When solving the nonprobabilistic reliable index, the upper and lower bounds of dam risk factor $X_{i}(i=1,2, \ldots, n)$ are set as $X_{i}^{u}$ and $X_{i}^{l}$, respectively, where $X_{i} \in X_{i}^{I}=\left[X_{i}^{l}, X_{i}^{u}\right]$. Assume that the performance function $g\left(X_{i}\right)$ is a continuous function of $X_{i}(i=1,2, \ldots, n)$, and the function value $Z$ is also an interval variable, where $Z \in Z^{I}=\left[Z^{l}, Z^{u}\right]$. These assumptions give

$$
\begin{aligned}
& Z^{l}=\min g\left(X_{1}, X_{2}, \ldots, X_{n}\right) X_{i} \in X_{i}^{I}\left[X_{i}^{l}, X_{i}^{u}\right], \\
& Z^{u}=\min g\left(X_{1}, X_{2}, \ldots, X_{n}\right) X_{i} \in X_{i}^{I}\left[X_{i}^{l}, X_{i}^{u}\right] .
\end{aligned}
$$

According to the calculation flow in the interval analysis method, $Z^{l}$ and $Z^{u}$ of the performance function are obtained, and the mean $Z^{c}$ and dispersion $Z^{r}$ are obtained by substituting equations (23) and (24) to equations (20) and (21). Finally, the nonprobabilistic index $\eta$ of dam reliability can be obtained from equation (22).

3.3. The Target Reliability Index of the Dam. Generally, a larger than one reliability index indicates that the dam structure is in a reliable state. Given this broad index value range, engineering projects prefer a specific value which is defined as the target reliability index of dam. In probabilistic and nonprobabilistic assessment, this value is regarded as the probabilistic target reliability index $\beta_{T}$ and the nonprobabilistic target reliability index $\eta_{T}$, respectively.

In this paper, the probabilistic target reliability index $\beta_{T}$ is based on SDJ21-78 Design Specification for Concrete Gravity Dams and SDJ20-78 Design Code for Hydraulic Concrete Structures. A comprehensive evaluation is performed based on both domestic and international technical standards and long-term accumulated engineering practices. Since there is not much literature on the value of $\eta_{T}$, this paper obtains nonprobabilistic target reliability index through the relationship between the nonprobabilistic and the probabilistic target reliability index, whose expression is 


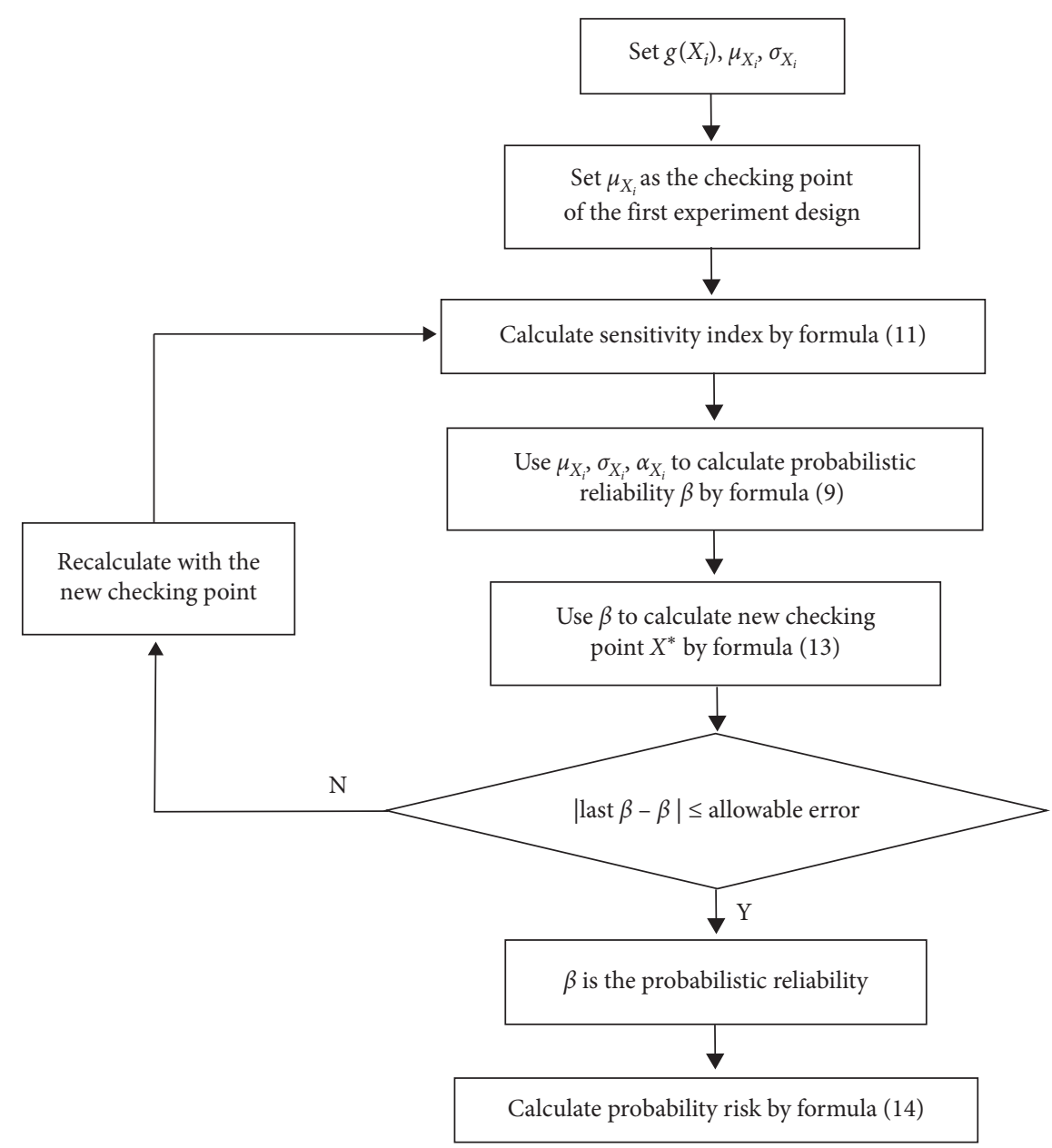

Figure 1: Flow chart of calculation steps of the probabilistic risk assessment model for dam.

$$
\eta_{T}=\frac{\beta_{T}}{k}
$$

where $\eta_{T}$ is the nonprobabilistic target reliability index; $\beta_{T}$ is the probabilistic target reliability index; and $k$ is the multiple of dispersion from standard deviation of the uncertainty factor, which gives $X_{i}^{r}=k \sigma_{X_{i}}$.

In equation (25), $\beta_{T}$ is obtained by the specification. Therefore, the key to determine $\eta_{T}$ is to determine a reasonable $k$ value. According to the change reasons of $k$ value, such as errors, uncertainties, and other factors caused by data processing, the nonprobabilistic target reliability index is selected as shown in Table 1 [38].

3.4. A Calculation Model of the Dam Reliability. The abovementioned contents provide a calculation method of the nonprobabilistic reliability index of dam reliability and the target reliability index of dam, respectively, but not the estimation of the risk probability of dam. Therefore, this section builds the nonprobabilistic assessment model for dam reliability evaluation based on the target reliability index of dam structure. According to the previous studies, when $\eta>\eta_{T}$, the dam is in a safe state and the probability of accident is 0 , namely, the risk probability $P_{f}$ is 0 ; when $\beta<1$ or $\eta<1$, the dam is in a state of failure and the probability of failure is $100 \%$, namely, the risk probability $P_{f}$ is $100 \%$. When $1<\beta<\beta_{T}$ or $1<\eta<\eta_{T}$, the probability of dam accident is uncertain. Therefore, in these cases, the probability of dam accident can only be determined by establishing the relationship between the nonprobabilistic reliability index and the risk probability of dam. The specific calculation expression is as follows:

$$
P_{f}= \begin{cases}0, & \eta>\eta_{T}, \\ \frac{\eta_{T}-\eta}{\eta_{T}-1}, & 1<\eta<\eta_{T}, \\ 100 \%, & \eta<1,\end{cases}
$$

where $P_{f}$ is the risk probability of dam, the risk probability here is different from the reliability expressed by probability and $\eta$ is the nonprobabilistic reliability index.

\section{The Combined Model for the Dam Risk Assessment}

The probabilistic method can be used to calculate the dam reliability only if sufficient information of dam impact 
TABle 1: Target reliability index $\beta_{T}$ and $\eta_{T}$ of dam.

\begin{tabular}{|c|c|c|c|c|c|}
\hline \multicolumn{3}{|c|}{ Structure safety grade } & I grade & II grade & III grade \\
\hline \multirow{4}{*}{ Damage type } & The first kind of failure & Probability target & 3.7 & 3.2 & 2.7 \\
\hline & The second kind of failure & Reliability index & 4.2 & 3.7 & 3.2 \\
\hline & The first kind of failure & Nonprobability & 1.3 & 1.2 & 1.1 \\
\hline & The second kind of failure & Target reliability index & 1.4 & 1.3 & 1.2 \\
\hline
\end{tabular}

factors is known; otherwise, the nonprobabilistic method can be used for evaluation when there is little or no information about dam structure impact factors. However, in dam engineering, the abundancy of information among factors is usually unbalanced; in some cases, some factors contain sufficient information, but these factors are not suitable to be described by a random model. In these cases, a single analysis method is not enough. Therefore, based on the probabilistic and nonprobabilistic assessment models for dam reliability evaluation, this section studies and proposes a combined model for dam risk assessment.

Since the probabilistic and nonprobabilistic assessment models for dam reliability evaluation are based on the performance function, the combined model cannot calculate the performance function simultaneously. Therefore, the idea of constructing a combination model by two levels of "probabilistic model before nonprobabilistic model" is proposed: at first, the bounded uncertainty factor is regarded as a general variable, and only the uncertainty of the probability factor is considered. Then, the calculation model of dam reliability calculates the reliability index function with uncertainty factor, providing an unspecified reliability index. The secondary performance function is latter established based on the probabilistic reliability index and probabilistic target reliability index. The uncertainty of the bounded uncertainty factor is considered in the secondary performance function to compute the nonprobabilistic reliability index and the risk probability of dam failure. The steps of the combined model are as follows:

(1) Assume that the dam performance function $Z^{(1)}$ which contains the probability factor and the bounded uncertainty factor is

$Z^{(1)}=g(X, Y)=g\left(X_{1}, X_{2}, \ldots, X_{n}, Y_{1}, Y_{2}, \ldots, Y_{m}\right)$,

where $X=\left\{X_{1}, X_{2}, \ldots, X_{n}\right\}$ is the probability factor vector; $Y=\left\{Y_{1}, Y_{2}, \ldots, Y_{m}\right\}$ is the bounded uncertainty factor vector; and $n$ and $m$ are the number of probability factors and bounded uncertainty factors, respectively.

(2) Consider the bounded uncertainty factor vector $Y$ in equation (27) as a general variable temporarily and calculate the probabilistic reliability index $\beta^{(1)}$ by the probabilistic model:

$$
\beta^{(1)}=\frac{\mu_{Z}}{\sigma_{Z}}=\beta(Y),
$$

where $\mu_{Z}$ and $\sigma_{Z}$ are the mean and standard deviation of the standard normal distribution performance function $Z$, respectively, and $\beta(Y)$ is a reliability index containing bounded uncertainty factor $Y$.

(3) Consider the uncertainty of bounded uncertainty factor $Y$ and the dam reliability conditions, and establish secondary performance function $Z^{(2)}$ as

$$
Z^{(2)}=\beta(Y)-\beta_{T},
$$

where $\beta_{T}$ is the target reliability index of the dam, and this index can be selected according to the safety grade and failure type of the dam.

Based on equation (29) and the nonprobabilistic assessment model for dam reliability evaluation, the nonprobabilistic reliability index is derived as follows:

$$
\eta^{(2)}=\frac{Z^{(2) c}}{Z^{(2) r}}=\frac{Z^{(2) u}+Z^{(2) l}}{Z^{(2) u}-Z^{(2) l}} \text {. }
$$

It can be seen from equation (30) that, when the extreme values obtained from the secondary performance function are all positive, the reliability index of the combined assessment model is larger than one. When the extreme values are all negative, the reliability index is negative. When the extreme values have one positive and one negative, the reliability index is between zero and one. Therefore, the risk probability portfolio of dam failure is given as follows:

$$
P_{f}= \begin{cases}0, & \eta^{(2)}>1, \\ \frac{1-\eta^{(2)}}{2}, & -1<\eta^{(2)}<1, \\ 100 \%, & \eta^{(2)}<-1 .\end{cases}
$$

\section{Case Study}

5.1. Project Profile. A gravity dam belongs to the secondclass project, and its foundation is horizontal. Its crest elevation is $223.0 \mathrm{~m}$ and its foundation level elevation is $153.0 \mathrm{~m}$. The normal water level of the reservoir is $220.0 \mathrm{~m}$, and the design flood level $(0.1 \%)$ is $221.0 \mathrm{~m}$. The water level of check flood $(0.02 \%)$ is $222.0 \mathrm{~m}$, and the dead water level is $185 \mathrm{~m}$. The conceptual model of the gravity dam is shown in Figure 2. 


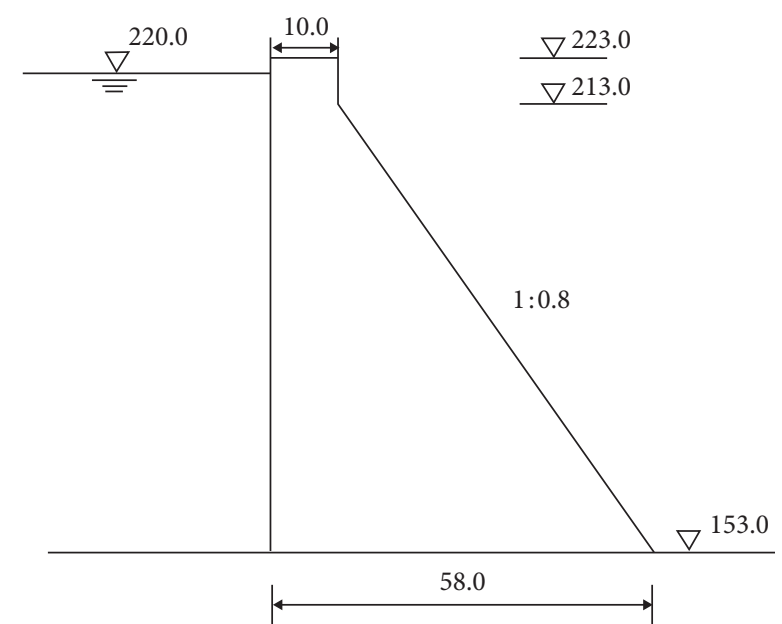

Figure 2: Typical cross section of nonoverflow dam section of a gravity dam (unit: $\mathrm{m}$ ).

In the engineering design of the dam, according to SDJ21-78 Design Specification for Concrete Gravity Dams and SDJ20-78 Design Code for Hydraulic Concrete Structures, the concrete bulk density $\gamma_{c}$ obeys normal distribution $N(23.5,0.47)$, the reduction coefficient of uplift pressure $\alpha$ obeys normal distribution $N(0.15,0.0375)$, and the shearbreak friction coefficient $f^{\prime}$ obeys normal distribution $N(1.1,0.18)$. The shear-break cohesion $c$ is set as a lognormal distribution, whose mean is $\mu_{c}=1.0 \mathrm{MPa}$ and the standard deviation is $\sigma_{c}=0.32 \mathrm{MPa}$. The design data show that the concrete bulk density $\gamma_{c}$, reduction coefficient of uplift pressure $\alpha$, shear-break friction coefficient $f^{\prime}$, and shear-break cohesion $c$ ranges from $[23.5,24],[0.3,0.4]$, $[1.0,1.3]$, and $[1.0,1.2]$ in turn. It is assumed that the four parameters are independent, as shown in Table 2.

5.2. Determination of the Performance Function. The performance function of the dam is mainly determined by the failure mode. In the reliability assessment of the gravity dam, the failure modes mainly include instability and insufficient strength. In this section, the reliability assessment in instability is taken as an example to study the dam reliability assessment. Other failure modes can be discussed in similar ways.

The instability along the foundation surface of the gravity dam is one of the main failure modes of the concrete gravity dam. Suppose the area of the sliding surface of the dam is $A$, the total vertical force on the sliding surface is $\sum W$ and the uplift pressure acting on the sliding surface is $U$. According to the literature [39], under normal conditions, the loads to be considered for the instability of gravity dam foundation surface are upstream water pressure, downstream water pressure, silting pressure, and wave pressure. According to the description of the project, the performance function of the dam stability along the dam foundation surface is as follows:

$$
\begin{aligned}
Z_{w 1} & =g_{w 1}\left(H_{1}, H_{2}, H_{3}, \alpha, c, f^{\prime}\right) \\
& =c A+f^{\prime}\left(\sum W-U\right)-\left(P_{w r 1}-P_{w r 2}+P_{s k}+P_{w k}\right),
\end{aligned}
$$

where $Z_{w 1}$ and $g_{w 1}\left(H_{1}, H_{2}, H_{3}, \alpha, c, f^{\prime}\right)$ represent the stability performance function of the dam along the foundation surface including the upstream water level $H_{1}$, downstream water level $H_{2}$, silting height $H_{3}$, uplift pressure coefficient $\alpha$, shear-break cohesion $c$, and friction coefficient $f^{\prime}$.

In this case, the upstream water level is chosen as the check water level, and the downstream water depth is zero. Therefore, the stability performance function of the project example along the dam foundation surface can be calculated from equation (32) as

$$
Z_{1}=\left(2140 \gamma_{c}-20010 \alpha-1725\right) f^{\prime}+58 c-23805 .
$$

\subsection{Calculation of the Assessment Model for Dam Reliability Evaluation}

\subsubsection{Calculation Based on the Probabilistic Risk Assessment} Model for Dams. Based on equations (9) and (11), after five iterations, the probabilistic reliability index $\beta=3.070785$ of the performance function is obtained. The iteration data are shown in Table 3, and the results of reliability index and risk probability data are shown in Table 4.

It can be seen from the results that the probability of instability along the dam foundation is $1.07 \times 10-3$. The results also show that the dam structure has good reliability and low-risk probability under specified conditions.

In the abovementioned calculation, the mean and variance of dam parameter variables are the original design values. Considering the volatility of the mean and variance due to the environmental impact, the following section mainly analyzes the relationship between the volatility of mean and variance and the risk probability of dam failure. Here, suppose that the fluctuation of mean and variance are controlled within $10 \%$.

Under the condition that the fluctuation of the mean and variance is controlled within $10 \%$ offset, the range of the mean and variance is shown in Table 5 during the interval analysis of dam reliability.

According to the detailed information of parameters listed in Table 5, the influence of the mean and variance fluctuation of dam variable parameters on probabilistic reliability index is analyzed from equations (29) and (30). We can conclude that the partial derivatives of dam probabilistic reliability index to the variance of each variable parameter is always less than zero, so the variance $\sigma_{X i}$ of dam probabilistic reliability index to each variable parameter is monotonically decreasing. The partial derivatives of the probabilistic reliability index to the mean $\mu_{\alpha}$ of the uplift pressure reduction coefficient are always less than zero, and the partial derivatives to the mean value of other variable parameters is always larger than zero. Therefore, the probabilistic reliability index has a monotonic decreasing relation to the mean value of the uplift pressure reduction coefficient and a monotonic increasing relation to the mean value of other parameters.

From the range of dam parameter variables in Table 5 and the research on the uncertainty of dam parameters in equations (29) and (30), along with the relations in equations (10) and (11), the maximum and minimum values of risk 
TABLe 2: Statistics of dam material parameters.

\begin{tabular}{lcccc}
\hline Parameter variable & Mean, $\mu$ & Standard deviation, $\sigma$ & Distribution type & Variable interval \\
\hline$\gamma_{c}$ & 23.5 & 0.47 & Normal distribution & \\
$\alpha$ & 0.15 & 0.0375 & Normal distribution & {$[23.5,24]$} \\
$f^{\prime}$ & 1.1 & 0.18 & Normal distribution & {$[0.3,0.4]$} \\
$c$ & 1.0 & 0.32 & Lognormal distribution & {$[1.0,1.3]$} \\
\hline
\end{tabular}

TABLE 3: Reliability iteration result statistics.

\begin{tabular}{lccc}
\hline Iteration times & $g$ & $\beta$ & $\left(\gamma_{c}, \alpha, f^{\prime}, c\right)$ \\
\hline 1 & 24171.75 & 3.035949 & $(23.301718,0.261803,0.561806,0.997735)$ \\
2 & 355.4752 & 3.071286 & $(23.394813,0.256261,0.549460,0.997648)$ \\
3 & -3.8288 & 3.070785 & $(23.397855,0.256080,0.549419,0.997664)$ \\
4 & $-4.08 E-04$ & 3.070785 & $(23.397887,0.256078,0.549418,0.997665)$ \\
5 & $-1.36 E-07$ & 3.070785 & $(23.397887,0.256078,0.549418,0.997665)$ \\
\hline
\end{tabular}

TABLE 4: Results of reliability index and risk probability data.

\begin{tabular}{lccc}
\hline Reliability index & Variable combination & Reliable index value & Risk rate \\
\hline$\beta$ & $(23.397887,0.256078,0.549418,0.997665)$ & 3.070785 & $1.07 \times 10-3$ \\
\hline
\end{tabular}

TABLE 5: Distribution parameters of random variables.

\begin{tabular}{lccc}
\hline Variable name & Mean & Variance & Distribution type \\
\hline$\gamma_{c}$ & $(21.15,25.85)$ & $(0.423,0.517)$ & Normal distribution \\
$\alpha$ & $(0.225,0.275)$ & $(0.03375,0.04125)$ & Normal distribution \\
$f^{\prime}$ & $(0.99,1.21)$ & $(0.162,0.198)$ & Normal distribution \\
$c(k P a)$ & $(0.9,1.1)$ & $(0.288,0.352)$ & Lognormal distribution \\
\hline
\end{tabular}

TABLE 6: Results of reliability index and failure probability.

\begin{tabular}{|c|c|c|c|c|c|}
\hline \multirow{2}{*}{$\begin{array}{l}\text { Reliability } \\
\text { index }\end{array}$} & \multicolumn{2}{|c|}{ Combination of probability factors } & \multirow{2}{*}{ Variable combination } & \multirow{2}{*}{$\begin{array}{l}\text { Reliable index } \\
\text { value }\end{array}$} & \multirow{2}{*}{$\begin{array}{c}\text { Failure } \\
\text { probability }\end{array}$} \\
\hline & Mean & Variance & & & \\
\hline$\beta_{\max }$ & $\begin{array}{c}\mu_{\gamma_{c}}=25.85 \mu_{\alpha}=0.225 \\
\mu_{f^{\prime}}=1.21 \mu_{c}=1.1\end{array}$ & $\begin{aligned} \sigma_{\gamma_{c}} & =0.376 \sigma_{\alpha}=0.03 \\
\sigma_{f^{\prime}} & =0.144 \sigma_{c}=0.256\end{aligned}$ & $\begin{array}{c}(25.744593,0.231274,0.487096 \\
1.097281)\end{array}$ & 4.473198 & $3.85 \times 10^{-6}$ \\
\hline$\beta_{\min }$ & $\begin{array}{c}\mu_{\gamma_{c}}=21.15 \mu_{\alpha}=0.275 \\
\mu_{f^{\prime}=0.99} \mu_{c}=0.9\end{array}$ & $\begin{array}{l}\sigma_{\gamma_{c}}=0.564 \sigma_{\alpha}=0.045 \\
\sigma_{f^{\prime}}=0.216 \sigma_{c}=0.384\end{array}$ & $\begin{array}{c}(21.062255,0.280223,0.629367 \\
0.898248)\end{array}$ & 1.833655 & $3.33 \times 10^{-2}$ \\
\hline
\end{tabular}

probability can be calculated. The results of the specific reliability and the corresponding failure probability values are shown in Table 6.

According to Table 6, the fluctuation of the mean and variance of the dam variable parameters has a great influence on the probabilistic reliability index of the structure and the dam reliability. Therefore, taking the above calculation flow as an example, we analyze the dam reliability controlled by the variable parameter volatility of the mean and variance under different values, so as to analyze the fluctuation between the mean and variance of the dam variable parameters and the risk probability, as shown in Figure 3 for details.

From Figure 3, when the uncertainty of the statistical characteristic quantity of dam variable parameters is small, the impact on the dam reliability is small. However, the upper limit of the dam reliability changes dramatically with the increase of uncertainty. Therefore, the volatility of the mean has a great influence on the results of the dam reliability.

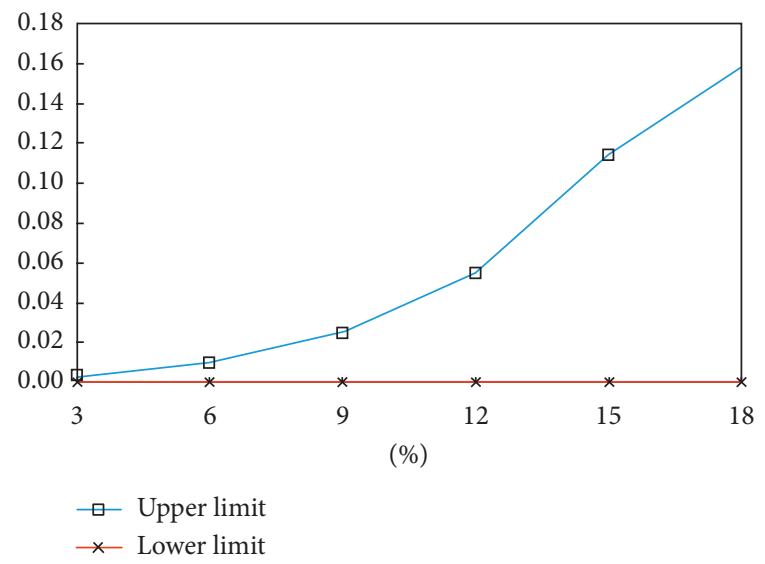

FIgURE 3: Relationship between the volatility of mean and variance and risk probability. 
5.3.2. Calculation Based on Nonprobabilistic Risk Assessment Model for Dams. From equation (29), solving the nonprobabilistic assessment model for dam reliability evaluation requires to solve the extreme value of dam performance function. The computational process is as follows:

$$
\begin{aligned}
& \frac{\partial Z_{1}}{\partial \gamma_{c}}=2140 f^{\prime} \\
& \frac{\partial Z_{1}}{\partial \alpha}=-20010 f^{\prime}, \\
& \frac{\partial Z_{1}}{\partial f^{\prime}}=2140 \gamma_{c}-20010 \alpha-1725, \\
& \frac{\partial Z_{1}}{\partial c}=58 .
\end{aligned}
$$

It can be seen from the analysis mentioned above that, in the parameter variable interval, the partial derivatives are always negative only when the performance function is partial derivate to $\alpha$, and the other partial derivatives are always positive. Therefore, in the parameter interval, the extreme values of the performance function are as follows.

When $\left(\gamma_{c}, \alpha, f^{\prime}, c\right)=(24,0.3,1.3,1.2)$, there is a maximum value:

$$
Z_{1}^{u}=Z_{1 \max }=32986.2,
$$

when $\left(\gamma_{c}, \alpha, f^{\prime}, c\right)=(23.5,0.4,1.0,1.0)$, there is a minimum value:

$$
Z_{1}^{l}=Z_{1 \min }=16814
$$

When the upstream water level rises to the check water level and the downstream water level is zero, the nonrisk probability reliability index of the dam is as follows:

$$
\eta_{1}=\frac{Z_{1}^{u}+Z_{1}^{l}}{Z_{1}^{u}-Z_{1}^{l}}=3.08
$$

As $\eta_{1}=3.08>\eta_{T}=1.3$, the dam is reliable and the risk probability of failure is very small.

5.3.3. Calculation Based on the Combined Model for Dams. According to equation (27), a risk assessment model for dam combining the probabilistic and the nonprobabilistic methods is established. Considering $\gamma_{c}, \alpha, f^{\prime}$, and $c$ as general variables, the expression of probabilistic reliability index is obtained from equation (28):

$$
\beta=\frac{2140 f^{\prime}\left(23.5-\gamma_{c}\right)-20010 f^{\prime}(0.15-\alpha)+1.1\left(2140 \gamma_{c}-20010 \alpha-1725\right)-23747}{\sqrt{1011634 f^{\prime 2}\left(23.5-\gamma_{c}\right)^{2}+563062.64 f^{\prime 2}(0.15-\alpha)^{2}+0.0324\left(2140 \gamma_{c}-20010 \alpha-1725\right)^{2}+344.47(1-c)^{2}}} .
$$

The dam has a II safety grade, and its stability target reliability index is 3.7. The secondary performance function is established as

$$
Z_{3}^{(2)}=\beta\left(\gamma_{c}, \alpha, f^{\prime}, c\right)-\beta_{T} .
$$

In the parameter interval, the maximum and the minimum of the secondary performance function are obtained as follows:

$$
\begin{aligned}
& Z_{\max }^{(2)}=209.3074, \\
& Z_{\min }^{(2)}=11.9701 .
\end{aligned}
$$

Then, the combined model index of the dam is

$$
\eta^{(2)}=\frac{Z_{\max }^{(2)}+Z_{\min }^{(2)}}{Z_{\max }^{(2)}-Z_{\min }^{(2)}}=1.1213
$$

Because $\eta^{(2)}=1.1213>1$, the risk probability of dam failure is very small.

\section{Conclusion}

This paper studies how to build the probabilistic and nonprobabilistic assessment model for dam reliability evaluation and also discusses and establishes a combined model of the dam considering the uncertainty of the parameters. The main research contents are as follows:
(1) In view of the shortcomings of the basic first-order second-moment method, the adjusted method is studied. Based on the adjusted first-order secondmoment method, the calculation model of dam reliability index is established. This paper discusses the relationship between the risk probability of dam failure and the probabilistic reliability index, constructs the probabilistic assessment model for dam reliability evaluation, and analyzes the influence of the mean and variance of the dam physical and mechanical parameters on the calculation result of the risk probability of dam failure.

(2) This paper analyzes the relationship between the bounded uncertainty of dam variable parameters and the nonprobabilistic reliability index and discusses the interval analysis method. The calculation model of nonprobabilistic reliability index is established. The relationship between the nonprobabilistic reliability index and the risk probability of dam failure are also studied. Accordingly, the nonprobabilistic assessment model for dam reliability evaluation is constructed.

(3) Considering the characteristics of probabilistic model and nonprobabilistic model for dam reliability evaluation, the fusion method of the two models is discussed, and the secondary performance 
function of dam failure is established. Based on the interval analysis method, the construction method of the combined model of dam reliability evaluation is studied.

\section{Data Availability}

The [Design parameters of the dam] data used to support the findings of this study are included within the article.

\section{Conflicts of Interest}

The authors declare that there are no conflicts of interest regarding the publication of this article.

\section{Acknowledgments}

This work was supported by the National Key R\&D Program of China (2018YFC0407104), the National Natural Science Foundation of China (Grant nos. 51739003, 51579085, 51779086, 51579086, 51379068, 51579083, and 51609074), the National Dam Safety Research Center (Grant no. CX2018Z38), the Fundamental Research Funds for the Central Public Welfare Research Institute (no. Y119002), Open Foundation of Changjiang Survey, Planning, Design and Research Co. Ltd (CX2019K01), Project funded by the Priority Academic Program Development of Jiangsu Higher Education Institutions (YS11001), the Special Project Fund of National Key Laboratory (20165042112), the Natural Science Foundation of Jiangsu Province (Grant no. BK20160872), the Key R\&D Program of Guangxi (AB17195074), and the Central University Basic Research Project (2017B11114 and 2018B25514).

\section{References}

[1] A. I. H. Malkawi, W. F. Hassan, and F. A. Abdulla, "Uncertainty and reliability analysis applied to slope stability," Structural Safety, vol. 22, no. 2, pp. 161-187, 2000.

[2] C. M. Kruger, A. C. Neto, and D. A. V. Kruger, "Structural reliability analysis and monitoring program applied to a roller-compacted concrete dam," in Proceedings of the 1st International Symposium on Life-Cycle Civil Engineering, LifeCycle Civil Engineering, Varenna, Italy, June 2008.

[3] O. Leszek, "The risk of dam stability loss," in Proceedings of the International Congress on Large Dam, Beijing,China, October 2000.

[4] O. Leszek, "The risk of dam instability," Express Water Resources \& Hydropower Information, vol. 22, no. 5, pp. 32-33, 2001.

[5] P. Liang, Z. Wu, J. Chen, Y. Li, and H. Zhang, "Reliability analysis of slope stability based on generalized feasible direction method," Rock and Soil Mechanics, vol. 34, no. 6, pp. 1753-1759+1785, 2013.

[6] Z. Ma, "Study on reliability of horizontal displacement at top of gravity dam based on checking point method," Urban Roads Bridges \& Flood Control, no. 11, pp. 150-153, 2013.

[7] X. Zheng, Q. Li, K. Xu, and L. Geng, "Calculation method on probability of tailings dam failure caused by dam slope instability considering correlation of variables," Journal of Safety Science and Technology, vol. 2, no. 11, pp. 23-27, 2015.
[8] H.-q. Liu, F. Xiao, X. Yang et al., "Dam reliability analysis method based on FEM-SVM," Water Resources and Power, no. 10, pp. 43-45, 2015.

[9] G. Wang, Z. Ma, J. Qin, and Q. Lan, "Reliability analysis on stabtility of gravity dam foundation over multiple sliding planes using PSO," Journal of Hydraulic Engineering, vol. 47, no. 2, pp. 219-228, 2016.

[10] G. Wang, J. Qin, and L. Guan, "Reliability analysis on stability of gravity dam foundation over multiple sliding planes based on GA," Chinese Journal of Rock Mechanics and Engineering, no. S1, pp. 3153-3161, 2016.

[11] Z. Lai, X. Chang, Y. Cheng et al., "Analysis of anti-sliding stability in deep foundation of guanyingyan gravity dam based on partial coefficient FEM," Water Power, vol. 43, no. 1, pp. 30-34, 2017.

[12] M. A. Hariri-Ardebili, "Analytical failure probability model for generic gravity dam classes," Proceedings of the Institution of Mechanical Engineers, Part O: Journal of Risk and Reliability, vol. 231, no. 5, pp. 546-557, 2017.

[13] I. Ellishakoff, "Essay on uncertainties in elastic and viscoelastic structures from A M Freudenthal's criticisms to modern convex modeling," Computersestructures, vol. 56, no. 6, pp. 871-895, 1995.

[14] B.-H. Yakov, "A non-probabilistic concept of reliability," Structural Safety, vol. 14, no. 4, pp. 227-245, 1994.

[15] I. Elishakoff, "Discussion on: a non-probabilistic concept of reliability," Structural Safety, vol. 17, no. 3, pp. 195-199, 1995.

[16] B.-H. Yakov, "A non-probabilistic measure of reliability of linear systems based on expansion of convex models," Structural Safety, vol. 17, no. 2, pp. 91-109, 1995.

[17] C. P. Pantelides and S. Ganzerli, "Design of trusses under uncertain loads using convex models," Journal of Structural Engineering, vol. 124, no. 3, pp. 318-329, 1998.

[18] S. Ganzerli and C. P. Pantelides, "Load and resistance convex models for optimum design," Structural Optimization, vol. 17, no. 2, pp. 259-268, 1999.

[19] M. Yuan, T. Lu, B. Xu, and B. Xu, "Reliability calculation of RCC gravity dam based on non-probabilistic method," Journal of China Three Gorges University (Natural Sciences), no. 6, pp. 29-33, 2013.

[20] Z. Qingfei, A New Type of Non-probabilistic Convex Model and its Corresponding Structural Uncertainty Analysis Technique, Hunan University, Hunan, China, 2013.

[21] J. Liu, M. Wang, and Q. Qing, "A new structural nonprobabilistic reliability analysis method," Chinese Journal of Solid Mechanics, no. 4, pp. 378-383, 2014.

[22] G. Su and J. Hao, "Gaussian process based dynamic response surface method of non-probabilistic reliability analysis for complicated engineering structure," Journal of Basic Science and Engineering, no. 4, pp. 750-762, 2015.

[23] B. Liang, Study on Stability and Reliability Analysis Method of Surrounding Rock and Support Structure of Tunnel, Hunan University, Hunan, China, 2015.

[24] Bi Rengui, An Uncertain Convex Set Model and a Nonprobabilistic Reliability Analysis Method. Hunan, Hunan University, Hunan, China, 2015.

[25] Y. Yun, J. Chen, and H. Cao, "Non-probabilistic reliability analysis on resonance of thermal-structural coupling of a beam based on improved Kriging," Journal of Harbin Institute of Technology, no. 10, pp. 131-136, 2016.

[26] P.-x. Zheng and M. Chen, "A response surface method based on SVM for dam reliability analysis," China Rural Water and Hydropower, no. 12, pp. 135-137, 2016. 
[27] M. Sun, X. Guo, M. Wang, and J. Wang, "Study on nonprobabilistic reliability of tunnel based on quadratic orthogonal test," China Civil Engineering Journal, no. S2, pp. 105-111, 2017.

[28] X. Li, Time-dependent Reliability Study of Crane Jib Structure Based on Interval Non-probabilistic Model, Taiyuan University of Science and Technology, Taiyuan, China, 2017.

[29] Y. Li, Robust Reliability Theory and Optimization Methods, Dalian University of Technology, Dalian, China, 2006.

[30] X. Qiao, On Reliability Analysis and Optimization of Uncertain Structures, Xidian University, Xi'an, China, 2009.

[31] B. Xu, R. Pang, and Y. Zhou, "Verification of stochastic seismic analysis method and seismic performance evaluation based on multi-indices for high CFRDs," Engineering Geology, vol. 264, Article ID 105412, 2020.

[32] R. Pang, B. Xu, D. Zou, and X. Kong, "Stochastic seismic performance assessment of high CFRDs based on generalized probability density evolution method," Computers and Geotechnics, vol. 97, pp. 233-245, 2018.

[33] W.-h. Mo, "HL-RF Method of gear reliability calculation," Journal of Hubei Industrial Polytechnic, no. 4, pp. 102-103, 2013.

[34] Y. Zhang, Y. Xu, Y. Zheng et al., "Multiobjective optimization design and experimental investigation on the axial flow pump with orthogonal test approach," Complexity, vol. 2019, Article ID 1467565, 14 pages, 2019.

[35] Y. Ben-Haim, Robust Reliability in the Mechancial Sciences, Springer-Verlag, Berlin, China, 1996.

[36] Y. Ben-Haim, "Robust reliability of structures," Advances in Applied Mechanics, vol. 33, pp. 1-41, 1997.

[37] Y. Li, X. Lu, L. Gan, Bo Chen, and H. Zhao, "Study on safety of cutoff wall of earth-rock cofferdam based on interval analysis," Water Resources and Power, no. 03, pp. 111-114, 2016.

[38] Y. Peng, Study on Risk Evaluation Model and Early-Warning Index of Concrete Gravity Dam, Hohai University, Hohai, China, 2008.

[39] C. Shen, S. Wang, Y. Lin et al., Hydraulic Structure, Water Resources and Hydropower Press, Beijing, China, 2008. 